\section{IN BRIEF}

\section{$\Rightarrow$ RHEUMATOID ARTHRITIS}

\section{Serum proteome altered in pre-RA}

Researchers have identified a pre-clinical proteomic signature that can predict future onset of rheumatoid arthritis (RA) in at-risk individuals (area under the curve 0.913, accuracy 91.2\%). The signature was developed by analysing serum samples from a cohort of first-degree relatives of indigenous North American patients with RA. Differences in the serum proteome between those who progressed to develop RA and the other at-risk individuals were present years before disease onset (median 30.9 months), irrespective of ACPA status.

ORIGINAL ARTICLE O'Neil, L. J. et al. A serum protein signature is associated with rheumatoid arthritis development. Arthritis Rheumatol. https://doi.org/10.1002/ art.41483(2020)

\section{EPIDEMIOLOGY}

\section{Cardiovascular risk underestimated in DISH}

In a cohort of 45 patients with diffuse idiopathic skeletal hyperostosis (DISH) without previously known cardiovascular disease (CVD), the actual extent of cardiovascular events over a 10-year period was higher than predicted by use of the Framingham risk score. Nearly $39 \%$ of the cohort had developed CVD after 10 years, higher than the anticipated $28.6 \%$. Notably, the retrospective analysis revealed that the incidence of myocardial infarction was higher among the patients with DISH than in a control group of 47 patients with osteoarthritis without DISH (24.4\% versus $4.3 \%, P=0.0055$ ).

ORIGINAL ARTICLE Glick, K. et al. Cardiovascular disease in diffuse idiopathic skeletal hyperostosis (DISH): from theory to reality - a 10-year follow-up study. Arthritis Res. Ther. 22, $190(2020)$

\section{$\Rightarrow$ SPONDYLOARTHRITIS}

\section{Secukinumab effective for $n r-a x S p A$}

In PREVENT, the first phase III study of the anti-IL-17A antibody secukinumab in patients with active non-radiographic axial spondyloarthritis ( $\mathrm{nr}-\mathrm{axSpA}$ ), the treatment improved signs and symptoms of the disease through 52 weeks with no new safety findings reported. The study met both its primary end points: the ASAS40 response rate was higher among patients who received secukinumab $150 \mathrm{mg}$ with loading doses than among those who received placebo at week 16 (41.5\% versus $29.2 \% ; P=0.0197)$, and was also higher in patients who received secukinumab $150 \mathrm{mg}$ without loading doses as compared with placebo at week 52 (39.8\% versus 19.9\%; $P<0.0021)$.

ORIGINAL ARTICLE Deodhar, A. et al. Secukinumab improves signs and symptoms of non-radiographic axial spondyloarthritis: primary results of a randomized controlled phase III study. Arthritis Rheumatol. https://doi.org/10.1002/art.41477 (2020)

\section{GOUT}

\section{Wearable devices track gout flares}

The findings of a 6-month, prospective pilot study suggest that the use of wearable activity trackers is a feasible way to collect data on the effect of patient-reported gout flares on physical activity and sleep. Of the 44 patients with physician-diagnosed gout enrolled in the study, 33 met the criteria for minimal wear time and flare reporting. Activity tracker data were available for $60.5 \%$ of all study days, and $75 \%$ of those days met the definition of compliant wear time. The data indicated that the mean daily step count was decreased during periods of gout flare compared with non-flare periods, although sleep did not differ.

ORIGINAL ARTICLE Elmagboul, N. et al. Physical activity measured using wearable activity tracking devices associated with gout flares. Arthritis Res. Ther. 22, 181 (2020)

\title{
MIS-C clinical guidance released amid race to define the condition
}

Multisystem inflammatory syndrome in children (MIS-C), which is also known as paediatric inflammatory multisystemic syndrome, has emerged as a rare but serious manifestation of severe acute respiratory syndrome coronavirus 2 (SARS-CoV-2) infection in children. In the face of the ongoing coronavirus disease 2019 (COVID-19) pandemic, the ACR has rapidly produced guidance to help clinicians provide the best possible care for children with COVID-19-related conditions, including MIS-C.

"Much like adults with COVID-19 pneumonia, children with complex medical problems can develop hyperinflammation in the setting of the acute, infectious phase of SARS-CoV-2 infections," explains Lauren Henderson, corresponding author on the new ACR guidance. "In addition, previously healthy children can develop MIS-C, a condition characterized by fever, inflammation and multiorgan dysfunction that occurs late in the course of SARS-CoV-2 infections."

The new guidance was compiled by a group of clinicians from a variety of subspecialties and covers several aspects of the diagnosis and treatment of MIS-C, including the importance of a broad differential diagnosis. "Children without life-threatening manifestations of MIS-C should undergo a diagnostic evaluation for other causes of the presentation (infectious and non-infectious), which may be more common than MIS-C," suggests Henderson.

According to two studies just published in The Journal of Clinical Investigation, despite having a broad clinical phenotype, MIS-C is distinct from both Kawasaki disease and macrophage activation syndrome (which share overlapping symptoms with MIS-C), and from COVID-19 itself. In both studies, specific patterns of cytokine expression were present in patients with MIS-C that differed from patterns in patients with the other conditions; results that are supported by preliminary data from in depth studies of the immunological landscape of MIS-C.

"Most children with MIS-C will need care from a multidisciplinary team that includes infectious disease specialists, rheumatologists, cardiologists and haematologists, among others. In particular, cardiology follow up is needed to ensure that cardiac function is normal and there is no coronary involvement," says Henderson.

The ACR guidance also covers the treatment of hyperinflammation associated with COVID-19 in children and recommends the use of immunomodulatory agents such as glucocorticoids, the IL-1 inhibitor anakinra or the IL-6 inhibitor tocilizumab. Glucocorticoids are also recommended as a first-line therapy for MIS-C in patients who are hospitalized, along with intravenous immunoglobulin.

"This guidance was developed over an expedited timeframe to help clinicians treating children during the current surge of COVID-19 cases in North America," states Henderson. "We view the guidance as a 'living document' and expect to update the content as further information becomes available about children with COVID-19."

\section{Joanna Clarke}

ORIGINAL ARTICLE Henderson, L. A. et al. American College of Rheumatology clinical guidance for pediatric patients with multisystem inflammatory syndrome in children (MIS-C) associated with SARS-CoV-2 and hyperinflammation in COVID-19. Version 1. Arthritis Rheumatol. https://doi.org/10.1002/ art.41454 (2020)

RELATED ARTICLES Diorio, C. et al. Multisystem inflammatory syndrome in children and COVID-19 are distinct presentations of SARS-CoV-2.J. Clin. Invest. https://doi.org/10.1172/JCI140970 (2020)| Lee, P. Y. et al. Distinct clinical and immunological features of SARS-COV-2-induced multisystem inflammatory syndrome in children. J. Clin. Invest. https://doi.org/10.1172/JCl141113 (2020)| Cavounidis, A. et al. Multisystem inflammatory syndrome in children: getting to the heart of the matter. Nat. Rev. Immunol. https://doi.org/10.1038/ s41577-020-0409-z (2020) 